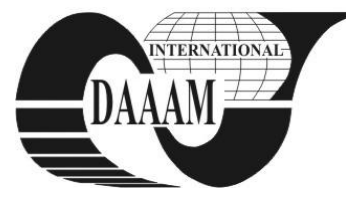

\title{
EVALUATION OF REAL ACCURACY OF AZIMUTH MEASUREMENT IN THE EXTERNAL ENVIRONMENT USING GNSS COMPASS
}

\author{
JILEK, T[omas] \& ZALUD, L[udek]
}

\begin{abstract}
This article deals with evaluation of accuracy of experimental azimuth measurement in external environment using the GNSS receiver with two antenna inputs. As a reference method for comparison of measurement results a trigonometric method is used. To allow usage of this method as a reference, this method is analyzed for the uncertainty of measurement. The experiment evaluates sets of repeated measuremennts for various values of azimuth. The result of the experiment is the knowledge of basic statistical parameters describing the azimuth measurement and the baseline length measurement.
\end{abstract}

Key words: GNSS, heading, GNSS compass, GPS compass, azimuth

\section{INTRODUCTION}

Angles of rotation of mobile device in all three axes are in a mobile robotics highly sought values, but their measurement is more difficult task that measuring position coordinates in the Cartesian coordinate system. The most problematic is the evalution of angle to true north - azimuth. In the external environment is possible to evaluate this angle by using a satellite navigation (GNSS), which is appear to be one of the most reliable method for automated measurement when the number of satellites is sufficient. A major advantage is the low sensitivity to magnetic fields and magnetically conductive objects located near of the site against use of magnetometer. The basic problem with this method is a time variability of accuracy, which depends mainly on the constellation of GNSS satellites. The measurement principle is based on evaluation of position difference of two antennas that are connected to a GNSS receiver with two antenna inputs. Because it is a differencial method of evaluation, the position offset error of both antennas is eliminated. For evaluation the accuracy of GNSS receivers in heading mode we used a simple, but relatively accurate trigonometric method.

Theoretical analysis of the azimuth measurement accuracy performed by GNSS receivers has been done before (Chansik, et al., 2011), but their reliability and validity is not known in a present. Therefore, we proposed a simple method that is used to verify the accuracy of azimuth measurement in the external environment. A result of our experiment is evaluation of key indicators of azimuth measurement accuracy and baseline length measurement accuracy using a Trimble BX982 device. These values are compared with parameters supplied by device manufacturer. In the following researches we want to establish a relationship which determines the required measuring time and measurement parameters to ensure the required accuracy in a specific constellation of GNSS satellites.

\section{REFERENCE METHOD}

For evaluation of baseline reference angle a trigonometric method was used. Using this method it is possible to evaluate the angle from the knowledge of constant baseline length and variable distance of moving endpoint of baseline from the reference point. In our experiment the baseline with length of 2 $\mathrm{m}$ was used. The measuring principle is shown in Figure No. 1.

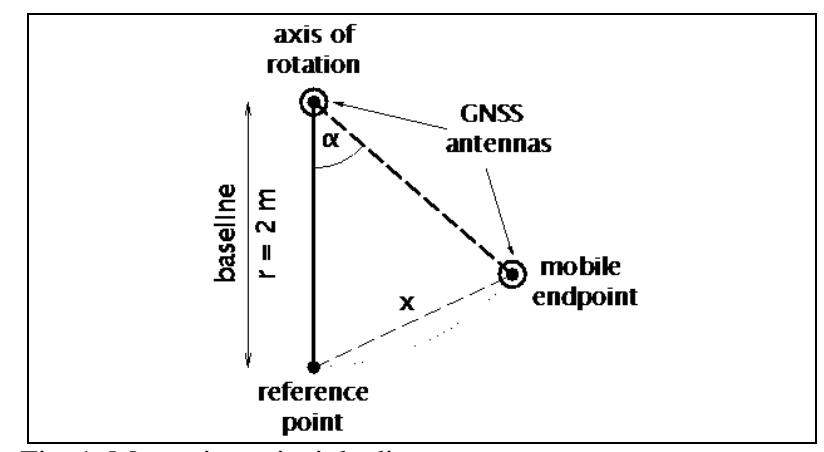

Fig. 1. Measuring principle diagram

Whereas that the resulting triangle is isosceles, applies to the $\alpha$ angle relationship:

$$
\alpha(x, r)=2 \arcsin \frac{x}{2 r}
$$

Type B measurement uncertainty of this indirect angle measurement depends on a measurement uncertainty of baseline length $r$ and the measurement uncertainty of a variable distance $x$ of moving endpoint of the baseline from the reference point. Resolution (graduation) of measuring device by which the distances were measured is $1 \mathrm{~mm}$. This device measures the baseline length and the distance of endpoints from the reference point. Type $\mathrm{B}$ measurement uncertainty of this indirect angle measurement can be determined from the relationship:

$$
u_{B \propto}(x, r)=\sqrt{\left(\frac{\partial \alpha(x, r)}{\partial x} u_{B x}\right)^{2}+\left(\frac{\partial \alpha(x, r)}{\partial r} u_{B r}\right)^{2}}
$$

where $u_{\mathrm{Bx}}$ is the uncertainty of variable distance $x$ and uncertainty $u_{\mathrm{Br}}$ is the uncertainty of baseline length. The calculation of these partial uncertainties is carried out according to relationships (3) and (4):

$$
\begin{aligned}
& u_{B x}=\frac{\Delta x_{\max }}{\chi} \\
& u_{B r}=\frac{\Delta r_{\max }}{\chi}
\end{aligned}
$$

These measurement uncertainties are based on values of the interval in which the measurement results may be found and on the basis of their distribution. After adjusting equation (2) and substituting relationships (3) and (4) we get:

$$
u_{B \alpha}(x, r)=\sqrt{\left(\frac{1}{r \sqrt{1-\frac{x^{2}}{4 r^{2}}}} \cdot \frac{\Delta x_{\max }}{\chi}\right)^{2}+\left(\frac{-x}{r^{2} \sqrt{1-\frac{x^{2}}{4 r^{2}}}} \cdot \frac{\Delta r_{\max }}{\chi}\right)^{2}}
$$


Due to fact that with increasing variable distance $x$ the type B measurement uncertainty of the $\alpha$ angle increases, it is advantageous angles from 90 degrees to 180 degrees from the reference point to evaluate as a complement to the angle $\alpha$, which is measured from the point, which is rotated from the reference angle of 180 degrees, as it is shown in Figure No. 2.

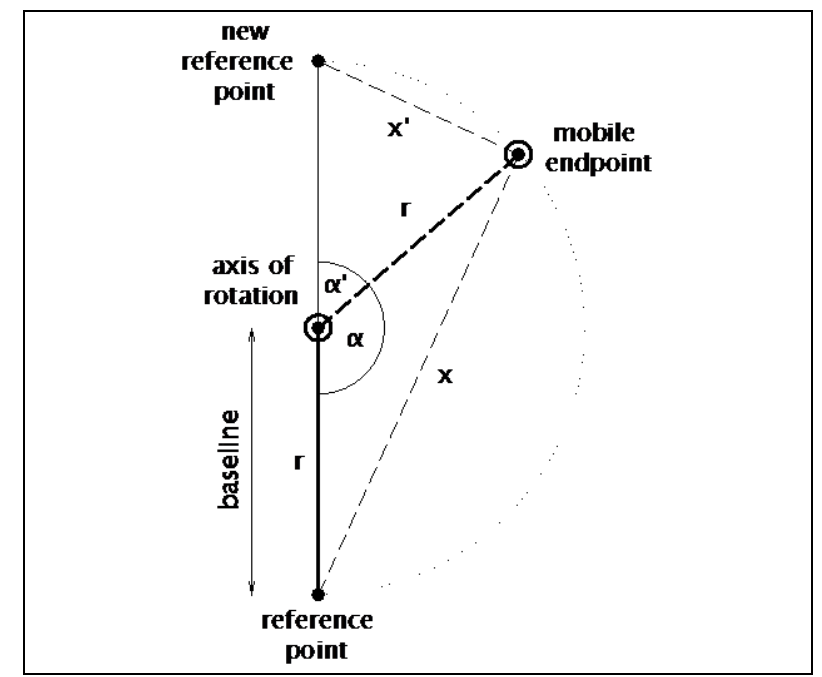

Fig. 2. Modified measuring principle diagram

In Figure No. 3 the dependence of type B measurement uncertainty on the measured angle for a described measuring method is shown.

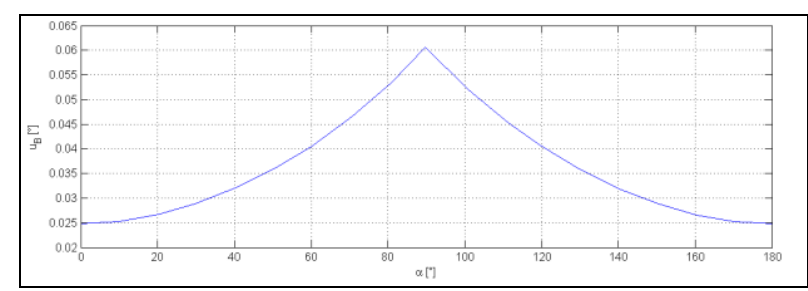

Fig. 3. Dependence of type B measurement uncertainty on the measured angle

\section{EXPERIMENTAL RESULTS}

All measurements were made on Trimble BX982 device with two antennas Trimble AG25. For calculation of the location from 14 to 16 satellites were used, PDOP value was in the range of 1.3 to 1.5 . The experiment evaluated the accuracy of azimuth of the baseline and the accuracy of its length. Angle step of measurement is approximately 10 degrees. For each adjusted value of azimuth 61 values in 61 seconds ( 1 value per second) were measured by the GNSS receiver.

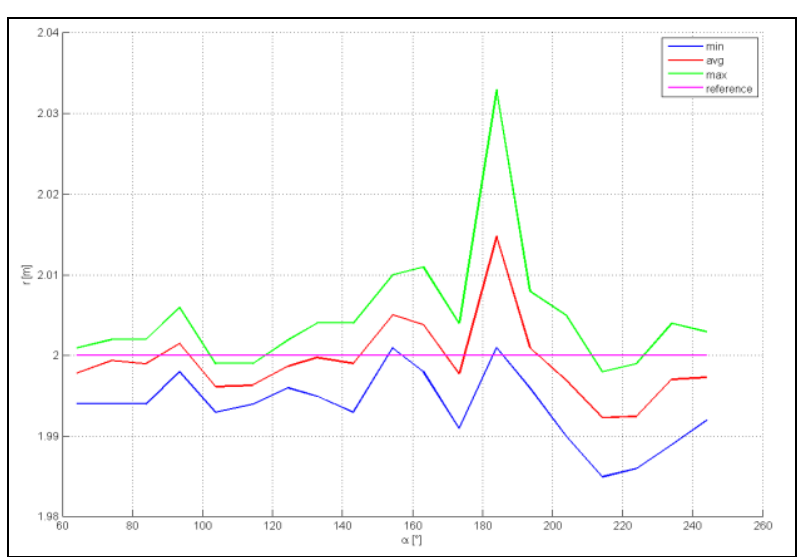

Fig. 4. Dependence of baseline length on azimuth

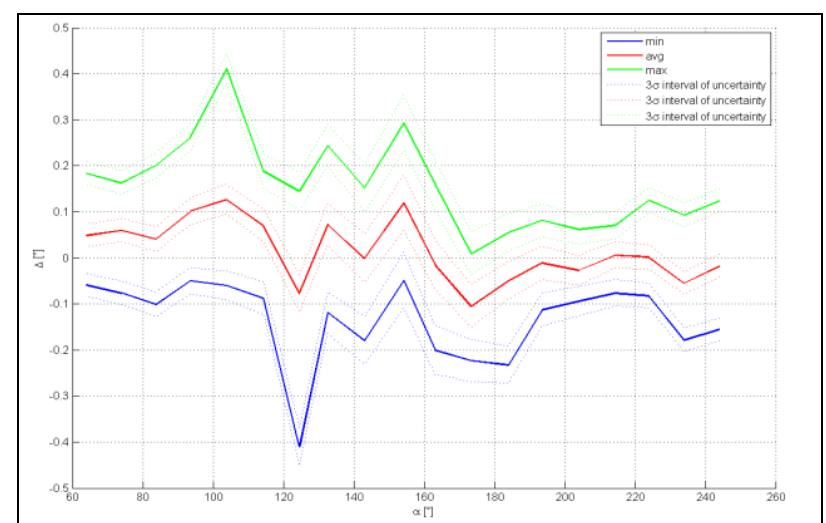

Fig. 5. Difference between the measured azimuth by the GNSS receiver and the angle calculated by the reference method

\section{CONCLUSION}

Based on the analysis of the reference method it was found that the chosen reference method may have a maximum uncertainty of measurement 0.021 degrees (one sigma interval for normal distribution) when the measurement uncertainty of dimensions is $0.29 \mathrm{~mm}$. The experiment showed that azimuth measurement error is not related with the baseline length measurement error. Baseline length is mostly constant and therefore its measurement error is determinable.

The main aim of the experiment was to verify the azimuth measurement accuracy of Trimble BX982 device. The device has reported accuracy by its manufacturer better that 0.09 degrees (one sigma interval). We find maximum standard deviation 0.10 degrees (at azimuth 124 degrees). For other values of azimuth the standard deviation is smaller than 0.09 degrees. Maximum deviation of calculated mean of the baseline length measurement from the reference measurement is $15 \mathrm{~mm}$. The mean of all baseline length measurements is $1.9993 \mathrm{~m}$, standard deviation is $5.5 \mathrm{~mm}$.

\section{ACKNOWLEDGEMENTS}

This work was supported by Operational Program Research and Development for Innovations; project CVVOZE - Center for Research and Utilization of Renewable Energy Sources, no. of contract CZ.1.05/2.1.00/01.0014.

\section{REFERENCES}

Chin-Lang, T.; Wei-Wen, K. (2006). Constrained total leastsquare solution for GPS compass attitude determination. Applied Mathematics and Computation, vol. 183, no. 1, pp. 106-118, ISSN 0096-3003

Chin-Lang, T.; Wei-Wen, K. (2006) Ill-conditioned GPS compass attitude determination using regularization approach. Applied Mathematics and Computation, vol. 183, no. 1, pp. 184-198, ISSN 0096-3003

Chansik, P.; Ilsun, K.; Gyu-In, J.; Jang, G. L. (1997). An error analysis of GPS compass. SICE '97. Proceedings of the 36th SICE Annual Conference. International Session Papers, pp. 1037-1042

Koura, Y.; Suzuki, H.; Ogawa, K.; Kamei, Y.; Nakamura, M. (2001). GPS COMPASS: A Low Cost GPS Direction Sensor of Two Antenna Type. Proceedings of the 14th International Technical Meeting of the Satellite Division of

Tu, C.-H.; Tu, K.Y.; Chang, F.-R.; Wang, L.-S. (1997). GPS compass: novel navigation equipment. Aerospace and Electronic Systems, IEEE Transactions on, vol. 33, no. 3, pp. 1063-1068, ISSN: 0018-9251

The Institute of Navigation (ION GPS 2001), Salt Lake City, UT, pp. 2700-2707 